\title{
Introducing SPA, "The Stellar Photometry Assistant"
}

\author{
J. Dalessio ${ }^{1,2,3}$, J. L. Provencal ${ }^{1,2} \&$ A. Kanaan ${ }^{4}$ \\ ${ }^{1}$ Delaware Asteroseismic Research Center, Mt. Cuba Astronomical Observatory, \\ Greenville, DE 19807 \\ 2 Department of Physics and Astronomy, The University of Delaware, 104 The \\ Green, 217 Sharp Laboratory, Newark, DE, 19716 \\ 3 Department of Astronomy, The Pennsylvania State University, 525 Davey Lab, \\ University Park, PA, 16802 \\ ${ }^{4}$ Departamento de Física, Universidade Federal de Santa Catarina, CP 476, \\ 88040-900, Florianópolis, SC, Brazil
}

\begin{abstract}
The Stellar Photometry Assistant, SPA, is a stand alone software package for time-series photometry reduction and analysis slated for an initial test release in spring 2008. The goal of SPA is to be simple, powerful, and intuitive. SPA was born out of complications in studying the pulsating DB white dwarf EC20058-5234 (QU Tel) due to the proximity of its nearby companions. SPA also addresses the Whole Earth Telescope's (WET) demand for large scale rapid data reduction from multiple sites. SPA is being developed in Matlab by the Delaware Asteroseismologic Research Center (DARC) in collaboration with the University of Delaware and the Mount Cuba Astronomical Observatory. The need for SPA is addressed, and key features of the program are listed and discussed.
\end{abstract}

\section{The Need for SPA}

QU Tel is a prime candidate for obtaining a $\dot{P}$ due to its rapid cooling rate and stable modes (Koen et. al. 1995). However, there are two nearby companions, one of which lies well within efficient aperture sizes. Due to the required precision to obtain a $\dot{P}$, combined with the general goal of reducing noise, more advanced data reduction techniques such as those described in Alard \& Lupton (1998) and Stetson (1987) may be more suitable than traditional aperture photometry. IRAF is capable of such methods, but the complexity of these 
methods using IRAF can be challenging to inexperienced users. While SPA is currently only capable of aperture photometry, the modular structure of SPA will allow future implementations of more powerful methods. Meanwhile, the WET obtains large sets of photometric data for several objects semi-annually. The large quantity of data, and the variety of observing sites can make data reduction challenging and tedious. During a typical WET observation, multiple users participate in data reduction around the clock. As data is analysed, time critical decisions are made selecting targets and coordinating observations. This makes rapid data reduction a necessity. A tool like SPA would ideally lessen the manpower required at headquarters for a WET observation. This would ease operation of the WET financially.

\section{Program Features}

\section{An Intuitive Interface}

SPA features a graphical user interface with tool tips and help menus. SPA has been designed with the hope that users new to time-series photometry will find it easy to use while advanced users will not suffer from a lack power. Users first build or load parameter files (mentioned below). They then select an entire directory contents or specific files to be processed. If calibration files are selected they are applied in a user specified manner. The structure of the interface is, however, constantly under revision.

\section{Smart Star Finding}

SPA will only require user entry of star pixel positions from one image per filter. SPA will build a "star orientation file" containing these positions and calculate relative object intensities. SPA can then automatically acquire/reacquire the objects for any full or partial image of this field when that "star orientation file" is selected. This database can be used for other telescopes, even when the field of view is substantially different (as long as the field of view is specified). This allows large jumps in star positions to be easily handled with no interruption as well as lessening the amount of user input required for reduction of multiple observations of the same field (as in the case of the WET). This method will allow for both translational and rotational variance from the previously known object pixel coordinates. Currently SPA searches for a local maximum near the last known position of the object and centers with a simple model PSF. More advanced methods are still in development and automatic star finding may not be included in the initial test release. 


\section{FITS Header Flexibility}

Fits headers vary site to site and this presents difficulties reducing data for large scale collaborations such as the WET. SPA allows users to enter location information into a database. This includes information about header formatting. When processing a data set SPA can either detect the location from the fits headers automatically or the location can be selected from the database. This eliminates the need for managing and editing fits headers.

\section{Portability}

SPA will be available on a variety of platforms. The goal will be to have a free standing program with a simple single file distribution (including the Matlab Runtime Component). Functionality will be identical on all platforms. Public availability of the source code is under discussion. While contribution from the community will be valuable, it may be advantageous to restrict source distribution in order to be able to simplify supporting the software.

\section{Standardized Output}

SPA also builds a "SPA output file" (*.SPA) including the photometry parameters used, calibration information, various data from the light curve (raw counts, sky counts, etc.), processing logs, along with any analysis performed on the data. These files are exchangeable and can be loaded by another SPA user. SPA can export data from these files into an ASCII table. SPA also allows quick visualization of data contained in these SPA files.

\section{Analysis Tools}

Currently, the only analysis tool built into SPA, besides plotting, is the discreet Fourier transform (DFT). However, additional tools may be added before the initial release, such as pre-whitening. 


\section{Testing the Effectiveness of SPA}

IRAF scripts developed by Kanaan et. al. (2002) are being used to test the reliability and effectiveness of SPA. Simple aperture photometry produces similar light curves. Differences in the light curves are marginal and are likely due to variations in the methods chosen to round pixels or center an object. At first glance SPA seems substantially better at tracking stars in crowded fields. A more rigorous comparison is planned prior to the initial release.

Acknowledgments. Special thanks to the WET team and the Mount Cuba Astronomical Observatory for their support.

\section{References}

Alard, C., \& Lupton, R. H. 1998, ApJ, 503, 325

Kanaan, A., Kepler, S. O., \& Winget, D. E. 2002, A\&A, 389, 896

Koen, C., O‘Donoghuee, D., Stobie, R. S., et al. 1995, MNRAS, 277, 913

Stetson, P. B. 1987, PASP, 99, 191

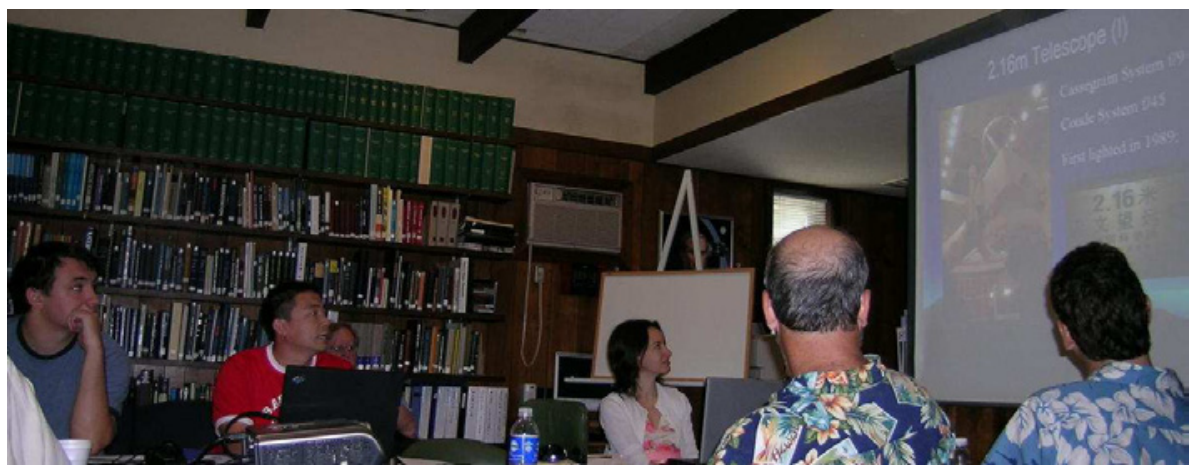

J. Xiaojun informs the WET about the BAO telescopes. 
